\title{
Dirac fermion, cosmological event horizons, and quantum entanglement
}

\author{
Sourav Bhattacharya, ${ }^{*}$ Shankhadeep Chakrabortty, ${ }^{\dagger}$ and Shivang Goyal ${ }^{*}$ \\ Department of Physics, Indian Institute of Technology Ropar, Rupnagar, Punjab 140 001, India
}

(Received 16 January 2020; accepted 6 April 2020; published 27 April 2020)

\begin{abstract}
We discuss the field quantization of a free massive Dirac fermion in the two causally disconnected static patches of the de Sitter spacetime, by using mode functions that are normalizable on the cosmological event horizon. Using this, we compute the entanglement entropy of the vacuum state corresponding to these two regions, for a given fermionic mode. Further extensions of this result to more general static spherically symmetric and stationary axisymmetric spacetimes are discussed. For the stationary axisymmetric Kerr-de Sitter spacetime in particular, the variations of the entanglement entropy with respect to various eigenvalues and spacetime parameters are depicted numerically. We also comment on such variations when instead we consider the nonextremal black hole event horizon of the same spacetime.
\end{abstract}

DOI: 10.1103/PhysRevD.101.085016

\section{INTRODUCTION}

The de Sitter spacetime is the simplest solution of the Einstein equation with a positive cosmological constant, $\Lambda$. It is maximally symmetric with isometry group $S O(4,1)$ in dimension 4. Its physical significance is chiefly twofold. First, owing to the observed accelerated expansion of the current Universe, there seems to be a strong possibility that our current Universe is endowed with a small but positive $\Lambda$, or some alternative form of the dark energy. Second, the observed high degree of spatial homogeneity and isotropy in all directions in the sky at large scales indicates that the very early Universe might also have gone through a phase of a rapid accelerated expansion, known as the inflation; see, e.g., Ref. [1] and references therein.

The positive $\Lambda$ is the simplest and phenomenologically a very successful model of the dark energy. Even though the exact nature/form of the dark energy is yet far from being well understood, it is reasonable to expect that the de Sitter spacetime would qualitatively model at least some salient features of any cosmological spacetime undergoing accelerated expansion. In particular, the highest degree of symmetry present in this spacetime makes many computations doable analytically.

There has been tremendous effort over decades to explore various aspects of quantum fields living in a de

\footnotetext{
sbhatta@iitrpr.ac.in

s.chakrabortty@iitrpr.ac.in

2017phz0003@iitrpr.ac.in
}

Published by the American Physical Society under the terms of the Creative Commons Attribution 4.0 International license. Further distribution of this work must maintain attribution to the author(s) and the published article's title, journal citation, and DOI. Funded by SCOAP.
Sitter universe. A complete review on this topic is far from the scope of this paper. We refer our reader to Refs. [2-4] for various aspects of particle creation and vacuum states in the cosmological de Sitter spacetime. See Ref. [5] and references therein for a study on the Schwinger effect in de Sitter. See, e.g., Refs. [6-11] for aspects of particle creation and thermal effects in the static de Sitter or de Sitter black hole spacetimes. Further, we refer our reader to, e.g., Refs. [12-14] (also references therein) for discussions on the late time nonperturbative infrared effects in the cosmological de Sitter spacetime. See also Refs. [15,16] for discussions on fermion-driven inflation.

A natural and interesting aspect of the de Sitter space is the relativistic quantum entanglement of fields, which is the focus of this paper. If we consider an "in" vacuum state in the cosmological de Sitter spacetime, due to the accelerated expansion, the state may evolve in the future to a different or "out" vacuum state, indicating particle pair production. Such pairs turn out to be entangled. On the other hand, due to the accelerated expansion, all parts of the de Sitter space cannot be causally connected. Quantum fields living in various causally disconnected parts of de Sitter can show a very nontrivial aspect of quantum entanglement. We refer our reader to Refs. [17-33] and references therein for a study of quantum field theoretic entanglement in the cosmological and hyperbolic coordinatization of de Sitter. We further refer our reader to Refs. [34-37] and references therein for a study of holographic aspects of de Sitter entanglement entropy.

The static coordinatization of de Sitter is interesting in the sense that the cosmological event horizon is explicitly "visible" in it and, second, it is explicitly time translational invariant (within the cosmological event horizon), e.g., Ref. [6]. The maximal analytic extension of the spacetime across this horizon shows, like that of the nonextremal 
black hole or the Rindler horizon, four causally disconnected spacetime regions, two of which are static, Sec. II. A quantum field living in these two regions possesses local vacua as well as some global vacuum defined with respect to the Kruskal null coordinates. It is thus interesting to address the issue of quantum entanglement between the quantum fields living in these two static regions, say $\mathrm{R}$ and $\mathrm{L}$. This issue was addressed recently in Ref. [38] for a scalar field, using the closed form mode functions, and the behavior of the entanglement entropy was shown to be similar to that of the Rindler spacetime.

In this work, we wish to do the same for the Dirac fermions. Precisely, using the closed form mode functions obtained in Sec. III for the static de Sitter coordinate, Eq. (1), we derive the R-L entanglement for the vacuum state of the Dirac field in Sec. IV. The result is found to be similar to that of the Rindler spacetime, found earlier in Refs. [39,40]. Such similarity follows from the universal Rindler-like behavior of the $t-r$ part of any (nonextremal) near-Killing horizon metric and the subsequent simplification of the mode functions. Such universality and simplification allow us to extend our result further to (a) the de Sitter horizon of a general static and spherically symmetric spacetime, such as the Schwarzschild-de Sitter, Sec. IV, and also to (b) stationary axisymmetric spacetime such as the Kerr-de Sitter, Sec. V. For the latter in particular, we numerically investigate the behavior of the entanglement entropy with respect to the variation of the energy, the angular momentum eigenvalues, and the spacetime mass and rotation parameters.

We shall work with the mostly negative signature of the metric in $3+1$ dimensions and will set $c=G=\hbar=1$ throughout.

\section{STATIC DE SITTER-A QUICK REVIEW}

The metric of the static patch of the de Sitter spacetime reads

$d s^{2}=\left(1-r^{2}\right) d t^{2}-\left(1-r^{2}\right)^{-1} d r^{2}-r^{2}\left(d \theta^{2}+\sin ^{2} \theta d \phi^{2}\right)$,

where the radial coordinate is made dimensionless via scaling by $H_{0}^{-1}=\sqrt{3 / \Lambda}$. The coordinate system is not well defined for $r \geq 1$. This corresponds to the fact that the timelike Killing vector field of Eq. (1) only exists in $0 \leq r<1$. The $r=1$ null hypersurface is the cosmological event horizon serving as the causal boundary of our Universe.

The singularity at $r=1$ of this metric can be removed by choosing Kruskal-like coordinates, which analytically continues the metric into the region $r \geq 1$, e.g., Ref. [6]. In terms of the Kruskal null coordinates $(\bar{u}, \bar{v})$, the metric reads

$$
d s^{2}=(1+r)^{2} d \bar{u} d \bar{v}-r^{2}\left(d \theta^{2}+\sin ^{2} \theta d \phi^{2}\right),
$$

where $r$ above as a function of $\bar{u}, \bar{v}$ is understood and

$$
\begin{gathered}
\bar{u}:=-e^{u}, \quad \bar{v}:=e^{-v} \\
u=t-r_{\star}, \quad v=t+r_{\star}, \quad r_{\star}=\frac{1}{2} \ln \left|\frac{1+r}{1-r}\right| .
\end{gathered}
$$

The tortoise coordinate $r_{\star}$ reaches $\infty$ as $r \rightarrow 1$. The metric Eq. (2) has no singularity at $r=1$.

The coordinate system described in Eqs. (2) and (3) covers the region $\mathrm{R}\left(0 \leq r_{R} \leq 1\right)$ of Fig. 1 . $\mathcal{C}^{ \pm}$are, respectively, the future and past segments of the cosmological event horizon. We have

$$
\bar{u}\left(\mathcal{C}^{+}\right)=0=\bar{v}\left(\mathcal{C}^{-}\right)
$$

and in general

$$
\bar{u} \leq 0, \quad \bar{v} \geq 0 \quad(\text { region } \mathrm{R})
$$

Likewise in region $\mathrm{L}$, we define

$$
\bar{u}:=e^{u}, \quad \bar{v}:=-e^{-v}
$$

so that

$$
\bar{u} \geq 0, \quad \bar{v} \leq 0 \quad(\text { region } \mathrm{L})
$$

with Eq. (4) still holding.

Regions I and II, being located at $r>1$, are not endowed with any timelike Killing vector field. We have

$$
\bar{u}>0, \quad \bar{v}>0 \quad(\text { region I }) \quad \bar{u}<0, \quad \bar{v}<0 \quad(\text { region II) }
$$

It is manifest from the orientations of $\mathcal{C}^{ \pm}$in Fig. 1 that the directions of the timelike Killing vector field, $\partial_{t}$, are opposite with respect to each other in $\mathrm{R}$ and $\mathrm{L}$. We shall

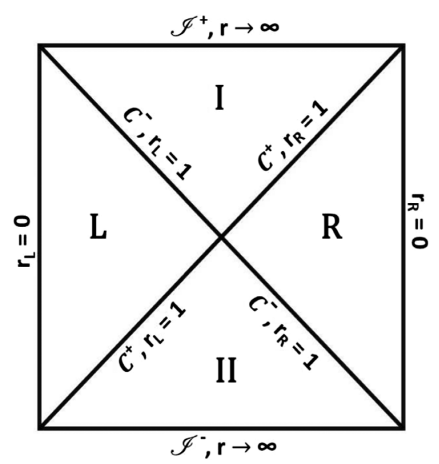

FIG. 1. The Penrose diagram of the de Sitter spacetime, found after the maximum analytic extension of Eq. (1). Each point of the above diagram is a 2 -sphere. $\mathcal{C}^{ \pm}$denote, respectively, the future and past cosmological event horizons. The causally disconnected static patches $\mathrm{R}$ and $\mathrm{L}$ are the regions of our concern. Region $\mathrm{L}$ is the time reversal of $\mathrm{R}$, and hence the timelike Killing vector field is taken to be past directed in $\mathrm{L}$. Regions I and II are nonstatic located in $r>1$. The infinities, $\mathcal{I}^{ \pm}$, are spacelike. 
accordingly take it to be future directed in $\mathrm{R}$, whereas it is past directed in L. The existence of $\mathcal{C}^{ \pm}$, just like the black hole, splits the spacetime into four causally disconnected wedges R, L, I, and II. For example, a particle initially in R can only cross $\mathcal{C}^{+}$to enter region I along a future directed trajectory, whereas it can only enter $\mathrm{R}$ from the contracting region $\mathrm{L}$ via $\mathcal{C}^{-}$.

Similar causally disconnected regions also show up across the cosmological event horizon of a general static spherically symmetric spacetime (e.g., the Schwarzschildde Sitter) and the stationary axisymmetric spacetime (e.g., the Kerr-de Sitter). For all of them, we shall investigate the quantum entanglement between regions $\mathrm{R}$ and $\mathrm{L}$ for a free massive Dirac field.

\section{DIRAC EQUATION IN THE STATIC DE SITTER SPACETIME}

We shall solve in this section for the four mode functions of a free massive Dirac field in a closed form, in the background of Eq. (1) for both regions R and L in Fig. 1. The latin indices appearing below will stand for the local Lorentz frame, whereas the greek indices will denote the curved spacetime.
The Dirac equation in the static de Sitter coordinates was previously studied in Refs. [7,41,42] (also references therein) using the Newman-Penrose formalism for spinors developed in Ref. [43]. See also Refs. [44,45] for discussions on choices of spacetime bases and group theoretic treatment of Dirac operators in static and time-dependent de Sitter coordinates.

The Dirac equation in a generally covariant form reads

$$
\left[i \gamma^{\mu} D_{\mu}-m_{0}\right] \Psi=0
$$

where $m_{0}$ is the rest mass of the field. $\gamma_{\mu}:=e_{\mu}{ }^{a} \gamma_{a}$, where $e_{\mu}{ }^{a}$ 's are the tetrad. The spin covariant derivative is defined as

$$
D_{\mu}:=\partial_{\mu}+\frac{1}{2} \omega_{\mu a b} \Sigma^{a b},
$$

where $\Sigma^{a b}=\left[\gamma^{a}, \gamma^{b}\right] / 4$ and the Ricci rotation coefficients $\omega$ 's are given by

$$
\omega_{\mu{ }_{b}}^{a}=-e_{b}{ }^{\nu}\left(\partial_{\mu} e^{a}{ }_{\nu}-\Gamma_{\mu \nu}^{\lambda} e_{\lambda}^{a}\right) .
$$

Following Ref. [46], we now define for Eq. (1)

$$
e_{a}^{\mu} \equiv\left(\begin{array}{cccc}
\left(1-r^{2}\right)^{-\frac{1}{2}} & 0 & 0 & 0 \\
0 & \left(1-r^{2}\right)^{\frac{1}{2}} \sin \theta \cos \phi & \left(1-r^{2}\right)^{\frac{1}{2}} \sin \theta \sin \phi & \left(1-r^{2}\right)^{\frac{1}{2}} \cos \theta \\
0 & r^{-1} \cos \theta \cos \phi & r^{-1} \cos \theta \sin \phi & -r^{-1} \sin \theta \\
0 & -(r \sin \theta)^{-1} \sin \phi & (r \sin \theta)^{-1} \cos \phi & 0
\end{array}\right)
$$

The Dirac equation is expanded to be

$$
\left[\left(1-r^{2}\right)^{-\frac{1}{2}}\left(\gamma^{0} \partial_{t}-\frac{r}{2} \vec{\gamma} \cdot \hat{r}\right)+\sqrt{1-r^{2}}(\vec{\gamma} \cdot \hat{r}) \partial_{r}-\left(\frac{1-\sqrt{1-r^{2}}}{r}\right) \vec{\gamma} \cdot \hat{r}+\frac{1}{r}\left(\vec{\gamma} \cdot \hat{\theta} \partial_{\theta}+\frac{\vec{\gamma} \cdot \hat{\phi}}{\sin \theta} \partial_{\phi}\right)\right] \Psi+i m_{0} \Psi=0,
$$

where $\hat{r}, \hat{\theta}, \hat{\phi}$ refer to the usual unit vectors in spherical polar coordinates and the gamma matrices are defined as

$$
\gamma^{0}=\left(\begin{array}{cc}
I & 0 \\
0 & -I
\end{array}\right), \quad \gamma^{i}=\left(\begin{array}{cc}
0 & \sigma^{i} \\
-\sigma^{i} & 0
\end{array}\right), \quad i=1,2,3 .
$$

Decomposing $\Psi$ as

$$
\Psi=\left(\begin{array}{l}
\Psi_{1} \\
\Psi_{2}
\end{array}\right)
$$

where $\Psi_{1}$ and $\Psi_{2}$ are each two component spinors, and using

$$
\left(\vec{\sigma} . \hat{\theta} \partial_{\theta}+\frac{\vec{\sigma} . \hat{\phi}}{\sin \theta} \partial_{\phi}\right)=-(\vec{\sigma} . \hat{r})\left(\hat{K}_{2}-1\right),
$$

where $\hat{K}_{2}=\vec{\sigma} \cdot \hat{L}+1$ is an eigenoperator of the spherical spinors, we find that Eq. (8) splits into two coupled equations, 


$$
\begin{aligned}
& \left(\left(1-r^{2}\right)^{-\frac{1}{2}} \partial_{t}+i m_{0}\right) \Psi_{1}+(\vec{\sigma} \cdot \hat{r})\left(-\frac{r\left(1-r^{2}\right)^{-\frac{1}{2}}}{2}+\sqrt{1-r^{2}} \partial_{r}+\frac{\sqrt{1-r^{2}}}{r}-\frac{\hat{K}_{2}}{r}\right) \Psi_{2}=0 \\
& \left(\left(1-r^{2}\right)^{-\frac{1}{2}} \partial_{t}-i m_{0}\right) \Psi_{2}+(\vec{\sigma} \cdot \hat{r})\left(-\frac{r\left(1-r^{2}\right)^{-\frac{1}{2}}}{2}+\sqrt{1-r^{2}} \partial_{r}+\frac{\sqrt{1-r^{2}}}{r}-\frac{\hat{K}_{2}}{r}\right) \Psi_{1}=0 .
\end{aligned}
$$

We recall that the spherical spin-1/2 harmonics, $\Omega_{j l m}$, are simultaneous eigenfunctions of $L^{2}, S^{2}, J^{2}, J_{z}$, given by [47]

$$
\Omega_{l+1 / 2, l, m}(\theta, \phi)=\left(\begin{array}{c}
C_{l m}^{+} Y_{l, m-1 / 2}(\theta, \phi) \\
C_{l m}^{-} Y_{l, m+1 / 2}(\theta, \phi)
\end{array}\right), \quad \Omega_{l-1 / 2, l, m}(\theta, \phi)=\left(\begin{array}{c}
-C_{l m}^{-} Y_{l, m-1 / 2}(\theta, \phi) \\
C_{l m}^{+} Y_{l, m+1 / 2}(\theta, \phi)
\end{array}\right),
$$

where

$$
C_{l m}^{ \pm}=\sqrt{\frac{l \pm m+1 / 2}{2 l+1}}
$$

They satisfy the orthonormality relations (with $j=l \pm 1 / 2$ )

$$
\int \sin \theta d \theta d \phi \Omega_{j, l, m}^{\dagger} \Omega_{j^{\prime}, l^{\prime}, m^{\prime}}=\delta_{j j^{\prime}} \delta_{l l^{\prime}} \delta_{m m^{\prime}}
$$

Further, we shall make use of the following easily verifiable properties:

$$
\begin{aligned}
(\vec{\sigma} \cdot \hat{r}) \Omega_{l+1 / 2, l, m} & =-\Omega_{(l+1)-1 / 2, l+1, m}, \quad \hat{K}_{2} \Omega_{l+1 / 2, l, m}=(l+1) \Omega_{l+1 / 2, l, m} \\
(\vec{\sigma} \cdot \hat{r}) \Omega_{(l+1)-1 / 2, l+1, m} & =-\Omega_{l+1 / 2, l, m}, \quad \hat{K}_{2} \Omega_{(l+1)-1 / 2, l+1, m}=-(l+1) \Omega_{(l+1)-1 / 2, l+1, m} .
\end{aligned}
$$

\section{A. Mode functions in $R$ and $L$}

We now make the ansatz of variable separation,

$$
\Psi=\left(\begin{array}{c}
\Psi_{1} \\
\Psi_{2}
\end{array}\right)=e^{-i p t} \frac{\left(1-r^{2}\right)^{-\frac{1}{4}}}{r}\left(\begin{array}{c}
i g(r) \Omega_{l+1 / 2, l, m} \\
f(r) \Omega_{(l+1)-1 / 2, l+1, m}
\end{array}\right),
$$

with $p>0$. Note that the future directedness of the timelike Killing vector field in $\mathrm{R}$ guarantees the above mode to be positive frequency. However, since that vector field is past directed in L, the above mode will be negative frequency in $\mathrm{L}$.

We insert Eq. (12) into Eq. (9) and use Eq. (11) to obtain

$$
\begin{aligned}
& \left(1-r^{2}\right) f^{\prime}+\frac{(l+1)}{r} \sqrt{1-r^{2}} f=\left(p-m_{0} \sqrt{1-r^{2}}\right) g \\
& \left(1-r^{2}\right) g^{\prime}-\frac{(l+1)}{r} \sqrt{1-r^{2}} g=-\left(p+m_{0} \sqrt{1-r^{2}}\right) f
\end{aligned}
$$

where a prime denotes differentiation once with respect to $r$. Following Ref. [41], we now write

$$
f=\frac{R_{1}-R_{2}}{2}, \quad g=\frac{i\left(R_{1}+R_{2}\right)}{2} .
$$

Further defining

$$
R_{1}(r)=\left(1-r^{2}\right)^{-\frac{1}{4}} \sqrt{1-r}\left(f_{1}(r)-f_{2}(r)\right), \quad R_{2}(r)=\left(1-r^{2}\right)^{-\frac{1}{4}} \sqrt{1+r}\left(f_{1}(r)+f_{2}(r)\right),
$$

Eq. (13) can be expressed as 


$$
\begin{aligned}
& \left(1-r^{2}\right) f_{1}^{\prime}-\left(\frac{l+1}{r}-i m_{0} r\right) f_{1}=-\left[\frac{1}{2}+i p-(l+1)+i m_{0}\right] f_{2} \\
& \left(1-r^{2}\right) f_{2}^{\prime}+\left(\frac{l+1}{r}-i m_{0} r\right) f_{2}=-\left[\frac{1}{2}+i p+(l+1)-i m_{0}\right] f_{1} .
\end{aligned}
$$

Form the above equation, we get an uncoupled equation for $f_{1}$,

$$
\left(1-r^{2}\right)^{2} f_{1}^{\prime \prime}-2 r\left(1-r^{2}\right) f_{1}^{\prime}+\left[\left(1-r^{2}\right)\left(\frac{l+1}{r^{2}}+i m_{0}\right)-\left(\frac{l+1}{r}-i m_{0} r\right)^{2}+\left(l-i m_{0}+1\right)^{2}-\left(\frac{1}{2}+i p\right)^{2}\right] f_{1}=0 .
$$

The solution that is regular as $r \rightarrow 1$ is given by

$$
f_{1}(r)=r^{l+1}\left(1-r^{2}\right)^{\frac{1}{4}+\frac{i p}{2}} F\left(\frac{\left(2 l+2 i m_{0}+2 i p+5\right)}{4}, \frac{\left(2 l-2 i m_{0}+2 i p+3\right)}{4} ; \frac{3}{2}+i p ; 1-r^{2}\right) .
$$

We next plug $f_{1}$ into Eq. (16) to determine $f_{2}$. Using the identities 15.2.15, 15.2.24, and 15.2.17 of Ref. [48], we find

$$
F(a, b+1 ; c ; z)-\frac{a z}{c} F(a+1, b+1 ; c+1 ; z)-F(a, b ; c ; z)=0,
$$

which yields

$$
f_{2}(r)=-\frac{\left(2 l-2 i m_{0}+2 i p+3\right)}{\left(2 l-2 i m_{0}-2 i p+1\right)} r^{l+2}\left(1-r^{2}\right)^{\frac{1}{4}+\frac{i p}{2}} F\left(\frac{\left(2 l+2 i m_{0}+2 i p+5\right)}{4}, \frac{\left(2 l-2 i m_{0}+2 i p+7\right)}{4} ; \frac{3}{2}+i p ; 1-r^{2}\right) .
$$

Denoting now the mode function Eq. (12) by $\Psi_{p l m}^{R 1+}$, in terms of $f_{1}(r)$ and $f_{2}(r)$, we have

$$
\Psi_{p l m}^{R 1+}=e^{-i p t} \frac{\left(1-r^{2}\right)^{-\frac{1}{2}}}{2 r}\left(\begin{array}{c}
-\left[\sqrt{1-r}\left(f_{1}(r)-f_{2}(r)\right)+\sqrt{1+r}\left(f_{1}(r)+f_{2}(r)\right)\right] \Omega_{l+1 / 2, l, m} \\
{\left[\sqrt{1-r}\left(f_{1}(r)-f_{2}(r)\right)-\sqrt{1+r}\left(f_{1}(r)+f_{2}(r)\right)\right] \Omega_{(l+1)-1 / 2, l+1, m}}
\end{array}\right) .
$$

A few comments on the normalizability of the mode functions are in order here. In Refs. [7,41,42], the Dirac mode found is bounded at the origin, $r \rightarrow 0$, whereas they are seemingly not well defined at the cosmological horizon, $r=1$. Accordingly, the normalization integral for those modes must exclude the horizon. This problem is present for a massive scalar and a vector field as well, as pointed out in Ref. [8]. Our mode function, Eq. (20), on the other hand, is not well behaved as $r \rightarrow 0$, follows from the properties of the hypergeometric function [48]. Accordingly, its normalization integral must exclude $r=0$, which can be realized by considering modes which are localized near the horizon. To the best of our knowledge, a Dirac mode in the static de Sitter coordinate that is regular everywhere in $0 \leq r \leq 1$ is unknown. Such a problem may just be a coordinate artifact. The mode function we have taken will be appropriate to make the analytic continuation across the horizon in order to form the global modes, for our current purpose.

To find out the second positive frequency mode, we make the ansatz by "flipping" the angular part of the previous ansatz, Eq. (12),

$$
\Psi_{p l m}^{R 2+}=e^{-i p t} \frac{\left(1-r^{2}\right)^{-\frac{1}{4}}}{r}\left(\begin{array}{c}
i \zeta_{1}(r) \Omega_{(l+1)-1 / 2, l+1, m} \\
\zeta_{2}(r) \Omega_{l+1 / 2, l, m}
\end{array}\right),
$$

for which Eq. (9) takes the form

$$
\begin{aligned}
& \left(1-r^{2}\right) \zeta_{1}^{\prime}+\frac{(l+1)}{r} \sqrt{1-r^{2}} \zeta_{1}=-\left(p+m_{0} \sqrt{1-r^{2}}\right) \zeta_{2} \\
& \left(1-r^{2}\right) \zeta_{2}^{\prime}-\frac{(l+1)}{r} \sqrt{1-r^{2}} \zeta_{2}=\left(p-m_{0} \sqrt{1-r^{2}}\right) \zeta_{1} .
\end{aligned}
$$

Comparing the above with Eq. (13), we find that the equations become identical if we let $f \rightarrow-\zeta_{1}, g \rightarrow \zeta_{2}$, and $m_{0} \rightarrow-m_{0}$. Thus $\zeta_{1}(r)$ and $\zeta_{2}(r)$ are respectively determined by in Eq. (18) and Eq. (19), with $m_{0}$ replaced with $-m_{0}$.

Having thus determined the two positive frequency modes, we can obtain the negative frequency ones, simply found via the charge conjugation. Given a positive frequency mode $u_{p}$, its charge conjugation $v_{p}$ is defined as

$$
v_{p}=C\left(\bar{u}_{p}\right)^{T}=i \gamma^{2} u_{p}^{\star}
$$


Using the explicit expressions of the spherical spinors appearing below Eq. (9), it is easy to check that

$$
\left(i \sigma_{2}\right) \Omega_{l+1 / 2, l, m}^{*}=(-1)^{m+\frac{1}{2}} \Omega_{l+1 / 2, l,-m}, \quad \text { and } \quad\left(i \sigma_{2}\right) \Omega_{l-1 / 2, l, m}^{*}=(-1)^{m+\frac{3}{2}} \Omega_{l-1 / 2, l,-m} .
$$

Using this, from Eq. (20) and Eq. (21), we can at once find out the two negative frequency modes, $\Psi_{\omega l m}^{(1-)}$ and $\Psi_{\omega l m}^{(2-)}$.

For our purpose, we shall now demonstrate the near cosmological horizon limit of these mode functions. We first recall that

$$
{ }_{2} F_{1}(a, b, c, 0)=1 .
$$

Thus, Eq. (18) and Eq. (19) behave near the horizon as

$$
f_{1}(r \rightarrow 1) \approx\left(1-r^{2}\right)^{\frac{1}{4}+\frac{i p}{2}}, \quad f_{2}(r \rightarrow 1) \approx-\frac{\left(2 l-2 i m_{0}+2 i p+3\right)}{\left(2 l-2 i m_{0}-2 i p+1\right)}\left(1-r^{2}\right)^{\frac{1}{4}+\frac{i p}{2}} .
$$

Also in terms of the tortoise coordinates Eq. (3), we have as $r \rightarrow 1$

$$
\left(1-r^{2}\right)^{\frac{i p}{2}}=2^{\frac{i p}{2}} e^{-i p r_{\star}} .
$$

Using these, and after normalizing, we find from Eq. (20), Eq. (21), Eq. (22), and Eq. (23) the expected plane wave solutions near the horizon in the $\mathrm{R}$ region,

$$
\begin{array}{ll}
\Psi_{p l m}^{R 1+}=\frac{e^{-i p v_{R}}}{\sqrt{4 \pi} r\left(1-r^{2}\right)^{\frac{1}{4}}}\left(\begin{array}{c}
\Omega_{l+1 / 2, l, m} \\
\Omega_{(l+1)-1 / 2, l+1, m}
\end{array}\right), & \Psi_{p l m}^{R 2+}=\frac{e^{-i p v_{R}}}{\sqrt{4 \pi} r\left(1-r^{2}\right)^{\frac{1}{4}}}\left(\begin{array}{c}
\Omega_{(l+1)-1 / 2, l+1, m} \\
\Omega_{l+1 / 2, l, m}
\end{array}\right) \\
\Psi_{p l m}^{R 1-}=\frac{e^{i p v_{R}}}{\sqrt{4 \pi} r\left(1-r^{2}\right)^{\frac{1}{4}}}\left(\begin{array}{c}
\Omega_{(l+1)-1 / 2, l+1,-m} \\
\Omega_{l+1 / 2, l,-m}
\end{array}\right), & \Psi_{p l m}^{R 2-}=\frac{e^{i p v_{R}}}{\sqrt{4 \pi} r\left(1-r^{2}\right)^{\frac{1}{4}}}\left(\begin{array}{c}
\Omega_{l+1 / 2, l,-m} \\
\Omega_{(l+1)-1 / 2, l+1,-m}
\end{array}\right),
\end{array}
$$

where in order to distinguish the region we have explicitly put the level $\mathrm{R}$ and $v_{R}=t_{R}+r_{\star R}$. The massless plane wave behavior of the modes in the near-horizon limit is consistent with the universal property of spacetimes endowed with a Killing horizon, e.g., Ref. [43]. The overall factor $\sqrt{4 \pi}$ in the denominators appearing above is necessary to ensure the normalization, as described below.

The inner product between any two modes $\Psi_{i}$ and $\Psi_{j}$ in the background of Eq. (1) is defined as

$$
\left(\Psi_{i}, \Psi_{j}\right)=\int \frac{r^{2} d r}{\left(1-r^{2}\right)^{\frac{1}{2}}} \sin \theta d \theta d \phi \Psi_{i}^{\dagger} \Psi_{j}
$$

Since the mode functions we have found are assumed to be localized near $\mathcal{C}^{ \pm}$, and the inner product is independent of time, we may take the constant time hypersurface of the above integration to be infinitesimally close to either $\mathcal{C}^{ \pm}$in Fig. 1, where the modes take the simple form, Eq. (24). We choose our surface to be close to $\mathcal{C}^{+}$. We have, for example,

$$
\begin{aligned}
\left(\Psi_{p l m}^{R 1+}, \Psi_{p^{\prime} l^{\prime} m^{\prime}}^{R 1+}\right) & =\int_{r \rightarrow 1} \frac{r^{2} d r}{\left(1-r^{2}\right)^{\frac{1}{2}}} \sin \theta d \theta d \phi\left(\Psi_{p l m}^{R 1+}\right)^{\dagger} \Psi_{p^{\prime} l^{\prime} m^{\prime}}^{R 1+} \\
& =\delta j j^{\prime} \delta_{l l^{\prime}} \delta_{m m^{\prime}} \int_{-\infty}^{\infty} \frac{d v_{R}}{2 \pi} e^{-i\left(p-p^{\prime}\right) v_{R}} \\
& =\delta\left(p-p^{\prime}\right) \delta j j^{\prime} \delta_{l l^{\prime}} \delta_{m m^{\prime}}
\end{aligned}
$$

where we have used Eq. (3) and also Eq. (10).

Likewise, we can prove that the rest of the mode functions appearing in Eq. (24) are normalizable and the inner product between any two different modes is vanishing. Thus, Eq. (24) forms a complete orthonormal set in R.

The orthonormal modes in $\mathrm{L}$ can be found in exactly the same manner as described above. However, since the timelike Killing vector field is past directed there, the function $e^{i p t}$ should behave as a positive frequency. We shall write down the near-horizon forms only,

$$
\begin{array}{ll}
\Psi_{p l m}^{L 1+}=\frac{e^{i p v_{L}}}{\sqrt{4 \pi} r\left(1-r^{2}\right)^{\frac{1}{4}}}\left(\begin{array}{c}
\Omega_{l+1 / 2, l, m} \\
\Omega_{(l+1)-1 / 2, l+1, m}
\end{array}\right), & \Psi_{p l m}^{L 2+}=\frac{e^{i p v_{L}}}{\sqrt{4 \pi} r\left(1-r^{2}\right)^{\frac{1}{4}}}\left(\begin{array}{c}
\Omega_{(l+1)-1 / 2, l+1, m} \\
\Omega_{l+1 / 2, l, m}
\end{array}\right) \\
\Psi_{p l m}^{L 1-}=\frac{e^{-i p v_{L}}}{\sqrt{4 \pi} r\left(1-r^{2}\right)^{\frac{1}{4}}}\left(\begin{array}{c}
\Omega_{(l+1)-1 / 2, l+1,-m} \\
\Omega_{l+1 / 2, l,-m}
\end{array}\right), & \Psi_{p l m}^{L 2-}=\frac{e^{-i p v_{L}}}{\sqrt{4 \pi} r\left(1-r^{2}\right)^{\frac{1}{4}}}\left(\begin{array}{c}
\Omega_{l+1 / 2, l,-m} \\
\Omega_{(l+1)-1 / 2, l+1,-m}
\end{array}\right) .
\end{array}
$$


We can likewise find out the complete orthonormal set of $\mathrm{R}-\mathrm{L}$ modes characterized by the retarded coordinate $u$.

In the following, we shall use the causally disconnected near-horizon local mode functions, Eq. (24) and Eq. (25), to construct the global modes and will compute the Bogoliubov coefficients.

\section{GLOBAL MODES AND THE BOGOLIUBOV COEFFICIENTS}

We can construct the global modes having support in $R \cup L$ in a manner similar to that of the Rindler spacetime, e.g., Refs. [49,50]. We shall analytically continue an $\mathrm{R}$ mode to $\mathrm{L}$ and vice versa along a complex path, by using the Kruskal coordinates, well defined on $\mathcal{C}^{ \pm}$as discussed in Sec. II. Let us take the pair $\left(\Psi_{p l m}^{R 1+}, \Psi_{p l m}^{L 2-}\right)$ from Eq. (24) and Eq. (25). Since both of these modes behave as $e^{-i p v}$ near the horizon with $p>0$, we shall choose our complex path through the lower half-plane. The angular parts remain intact during this analytic continuation procedure.

In terms of the Kruskal coordinates, Sec. II, we have $\Psi_{p l m}^{R 1+} \sim(\bar{v})^{-i p}$ and $\Psi_{p l m}^{L 2-} \sim(-\bar{v})^{-i p}$. Thus, while continuing the latter mode through the lower half-plane onto the region $\mathrm{R}$, we must write $-\bar{v}=e^{-i \pi} \bar{v}$. Accordingly, we have $\Psi_{p l m}^{L 2-} \sim e^{-\pi p}(\bar{v})^{-i p}$. Thus, the linear combination,

$$
\Psi_{p l m}^{R 1+}+e^{-\pi p} \Psi_{p l m}^{L 2-},
$$

is analytic across the horizon, has support in $R \cup L$, and behaves as a global mode function. Normalizing, we denote this mode function as

$$
{ }_{G} \Psi_{p l m}^{(1)}=\frac{1}{\sqrt{2 \cosh p \pi}}\left(e^{\frac{\pi p}{2}} \Psi_{p l m}^{R 1+}+e^{-\frac{\pi p}{2}} \Psi_{p l m}^{L 2-}\right)
$$

Likewise, we find three other global mode functions to form an orthonormal set,

$$
\begin{aligned}
& { }_{G} \Psi_{p l m}^{(2)}=\frac{1}{\sqrt{2 \cosh p \pi}}\left(e^{\frac{\pi p}{2}} \Psi_{p l m}^{R 2+}+e^{-\frac{\pi p}{2}} \Psi_{p l m}^{L 1-}\right) \\
& { }_{G} \Psi_{p l m}^{(3)}=\frac{1}{\sqrt{2 \cosh p \pi}}\left(e^{\frac{\pi p}{2}} \Psi_{p l m}^{R 1-}-e^{-\frac{\pi p}{2}} \Psi_{p l m}^{L 2+}\right) \\
& { }_{G} \Psi_{p l m}^{(4)}=\frac{1}{\sqrt{2 \cosh p \pi}}\left(e^{\frac{\pi p}{2}} \Psi_{p l m}^{R 2-}-e^{-\frac{\pi p}{2}} \Psi_{p l m}^{L 1+}\right) .
\end{aligned}
$$

Clearly, there exists another set of global modes, ${ }_{G} \Psi_{p l m}^{(5)}$, ${ }_{G} \Psi_{p l m}^{(6)},{ }_{G} \Psi_{p l m}^{(7)},{ }_{G} \Psi_{p l m}^{(8)}$, found via the interchange $R \leftrightarrow L$ on the right-hand side of Eq. (26) and Eq. (27).

We quantize the Dirac field $\Psi$ now in $R \cup L$ in terms of the local modes, Eq. (24) and Eq. (25), as well as the global modes found above. In terms of the local modes, we have

$$
\Psi=\sum_{l m s} \int_{0}^{\infty} d p\left(c_{s_{p l m}^{R}}^{R} \Psi_{p l m}^{R s+}+d_{s_{p l m}}^{R \dagger} \Psi_{p l m}^{R s-}+c_{s_{p l m}}^{L} \Psi_{p l m}^{L s+}+d_{s_{p l m} \dagger}^{L \dagger} \Psi_{p l m}^{L s-}\right),
$$

where $s=1,2$. The operators satisfy the usual anticommutation relations

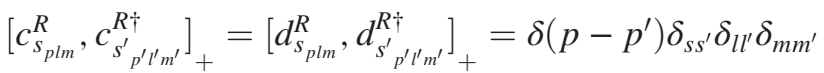

$$
\begin{aligned}
& {\left[c_{s_{p l m}}^{L}, c_{\left.s_{p^{\prime} l^{\prime} m^{\prime} m^{\prime}}^{L^{\prime}}\right]_{+}}^{L^{\prime}}=\left[d_{s_{p l m}}^{L}, d_{s_{p^{\prime} l^{\prime} m^{\prime}}^{\prime}}^{L^{\prime}}\right]_{+}=\delta\left(p-p^{\prime}\right) \delta_{s s^{\prime}} \delta_{l l^{\prime}} \delta_{m m^{\prime}},\right.}
\end{aligned}
$$

and all the other anticommutators vanish.

Likewise, in terms of the global modes, we have

$$
\begin{aligned}
\Psi= & \sum_{l m} \int_{0}^{\infty} d p\left(a_{1 p l m G} \Psi_{p l m}^{(1)}+a_{2 p l m G} \Psi_{p l m}^{(2)}+b_{1 p l m G}^{\dagger} \Psi_{p l m}^{(3)}+b_{2 p l m G}^{\dagger} \Psi_{p l m}^{(4)}\right. \\
& \left.+a_{3 p l m G} \Psi_{p l m}^{(5)}+a_{4 p l m G} \Psi_{p l m}^{(6)}+b_{3 p l m G}^{\dagger} \Psi_{p l m}^{(7)}+b_{4 p l m G}^{\dagger} \Psi_{p l m}^{(8)}\right) .
\end{aligned}
$$

Comparing Eq. (28) and Eq. (30) via Eq. (26) and Eq. (27), we obtain the Bogoliubov relations,

$$
\begin{array}{ll}
a_{1 p l m}=\frac{1}{\sqrt{2 \cosh \pi p}}\left(e^{\frac{\pi p}{2}} c_{1_{p l m}^{L}}^{L}-e^{-\frac{\pi p}{2}} d_{2_{p l-m} \dagger}^{R \dagger}\right), & a_{2 p l m}=\frac{1}{\sqrt{2 \cosh \pi p}}\left(e^{\frac{\pi p}{2}} c_{2_{p l m}^{L}}^{L}+e^{-\frac{\pi p}{2}} d_{1 p l-m}^{R \dagger}\right) \\
b_{1 p l m}^{\dagger}=\frac{1}{\sqrt{2 \cosh \pi p}}\left(e^{\frac{\pi p}{2}} d_{1_{p l-m} \dagger}^{\dagger \dagger}-e^{-\frac{\pi p}{2}} c_{2_{p l m}^{R}}^{R}\right), & b_{2 p l m}^{\dagger}=\frac{1}{\sqrt{2 \cosh \pi p}}\left(e^{\frac{\pi p}{2}} d_{2_{p l-m}^{\dagger}}^{L \dagger}+e^{-\frac{\pi p}{2}} c_{1_{p l m}}^{R}\right) .
\end{array}
$$


Another similar set is obtained by making the $R \leftrightarrow L$ interchange on the right-hand side of the above equations, for the operators $a_{3}, a_{4}, b_{3}, b_{4}$ appearing in Eq. (30). It is easy to check using Eq. (29) that the global operators satisfy the canonical anticommutation relations.

Since the above Bogoliubov coefficients are spacetime independent, the Bogoliubov relations are valid away from the horizons as well, even though we used the near-horizon forms of the modes to derive it. In other words, Eq. (27) will hold even if we are away from the horizon, with the local modes taking their nontrivial forms, e.g., Eq. (20).

Being equipped with these, we are now ready to compute the particle creation and the R-L entanglement for Eq. (1) for a free massive Dirac field.

\section{A. Entanglement entropy}

The local vacua $|0\rangle_{R},|0\rangle_{L}$ are defined as

$$
\begin{aligned}
& c_{s_{p l m}}^{R}|0\rangle_{R}=d_{s_{p l m}}^{R}|0\rangle_{R}=0, \\
& c_{s_{p l m}}^{L}|0\rangle_{L}=d_{s_{p l m}}^{L}|0\rangle_{L}=0 \quad(s=1,2),
\end{aligned}
$$

whereas the global vacuum is defined as

$$
a_{\sigma p l m}|0\rangle=b_{\sigma p l m}|0\rangle=0,
$$

where $\sigma=1,2,3,4$. Hereafter, we shall drop the indices $(p, l, m)$ on the operators for the sake of brevity.

From the Bogoliubov relations of the preceding section, it is clear that the operators $\left(a_{1}, a_{2}, b_{3}, b_{4}\right)$ and $\left(a_{3}, a_{4}, b_{1}, b_{2}\right)$ can be grouped into two sectors, anticommuting trivially. The global vacuum can therefore be decomposed as $|0\rangle=|0\rangle^{(1)} \otimes|0\rangle^{(2)}$, where $|0\rangle^{(1)}$ is annihilated by the first set of operators and $|0\rangle^{(2)}$ is annihilated by the second. Accordingly, we shall work with $|0\rangle^{(1)}$ only, for $|0\rangle^{(2)}$ will yield identical results.

The Bogoliubov relations imply a squeezed state relation of $|0\rangle^{(1)}$ with the local R-L vacua,

$$
|0\rangle^{(1)}=N \exp \left(\sum_{s, s^{\prime}=1,2} \xi_{s s^{\prime}} c_{s}^{L^{\dagger} \dagger} d_{s^{\prime}}^{R \dagger}\right)|0\rangle_{R}^{(1)} \otimes|0\rangle_{L}^{(1)},
$$

where $\xi_{s s^{\prime}}$ 's are four complex numbers, $d_{1}^{R}|0\rangle_{R}^{(1)}=$ $d_{2}^{R}|0\rangle_{R}^{(1)}=0$ and $c_{1}^{L}|0\rangle_{L}^{(1)}=c_{2}^{L}|0\rangle_{L}^{(1)}=0$ and $N$ is the normalization. Thus, we may further write

$$
\begin{gathered}
|0\rangle_{R}^{(1)}=\left|0_{d_{1}}\right\rangle_{R} \otimes\left|0_{d_{2}}\right\rangle_{R} \equiv\left|0_{d_{1}}, 0_{d_{2}}\right\rangle_{R}, \\
|0\rangle_{L}^{(1)}=\left|0_{c_{1}}\right\rangle_{L} \otimes\left|0_{c_{2}}\right\rangle_{L} \equiv\left|0_{c_{1}}, 0_{c_{2}}\right\rangle_{L},
\end{gathered}
$$

where $\left|0_{c_{1}}\right\rangle_{L},\left|0_{c_{2}}\right\rangle_{L}$ are annihilated by $c_{1}^{L}$ and $c_{2}^{L}$, respectively. Likewise, $\left|0_{d_{1}}\right\rangle_{R},\left|0_{d_{2}}\right\rangle_{R}$ are annihilated by $d_{1}^{R}$ and $d_{2}^{R}$, respectively. that

Since $|0\rangle^{(1)}$ is annihilated by $a_{1}, a_{2}, b_{3}, b_{4}$, it turns out

$$
\xi_{11}=\xi_{22}=0, \quad \xi_{12}=-\xi_{21}=e^{-\pi p} .
$$

We now write Eq. (34) as

$$
\begin{aligned}
|0\rangle^{(1)}= & N\left[1+e^{-\pi p}\left(-|0,1\rangle_{R}|1,0\rangle_{L}+|1,0\rangle_{R}|0,1\rangle_{L}\right)\right. \\
& \left.-e^{-2 \pi p}|1,1\rangle_{R}|1,1\rangle_{L}\right],
\end{aligned}
$$

where the normalization $N$ is given by

$$
N=\frac{1}{\left(1+e^{-2 \pi p}\right)} .
$$

As a check of consistency, we may compute the expectation value of the local number operator in the global vacuum. We find from Eq. (31), for example,

$$
{ }^{(1)}\left\langle 0\left|c_{1}^{L \dagger} c_{1}^{L}\right| 0\right\rangle^{(1)}=N^{2}\left(e^{-2 \pi p}+e^{-4 \pi p}\right)=\frac{1}{e^{2 \pi p}+1},
$$

showing fermionic "blackbody" distribution with temperature $1 / 2 \pi$.

The density operator corresponding to the global vacuum state, Eq. (37), is given by $\rho=|0\rangle^{(1)(1)}\langle 0|$. Tracing out now the states of the region inaccessible to us (say L), we find the reduced density operator $\rho_{R}=\operatorname{Tr}_{\mathrm{L}}\left(|0\rangle^{(1)(1)}\langle 0|\right)$. Its matrix representation is given by

$$
\rho_{R} \equiv N^{2}\left(\begin{array}{cccc}
1 & 0 & 0 & 0 \\
0 & e^{-2 \pi p} & 0 & 0 \\
0 & 0 & e^{-2 \pi p} & 0 \\
0 & 0 & 0 & e^{-4 \pi p}
\end{array}\right) .
$$

Finally, we obtain the entanglement entropy for a single mode characterized by $p$,

$S(p)=-\operatorname{Tr}\left(\rho_{R} \ln \rho_{R}\right)=2 \ln \left(1+e^{-2 \pi p}\right)+\frac{4 \pi p}{1+e^{2 \pi p}}$.

Note that the above result is identical to that of the Rindler spacetime $[39,40]$. This is not surprising, as the entanglement we are obtaining here is due to the existence of the R-L regions created by the bifurcation surface $\mathcal{C}^{ \pm}$in Fig. 1. Now, it is well known that the $t-r$ part of any nonextremal near-horizon geometry is similar to that of the Rindler. Thus, we expect a universality of the Bogoliubov coefficients, Eq. (31), for all nonextremal Killing horizons, be they black hole or cosmological, at least at the qualitative level. 
We shall further extend below the above result for both static spherically symmetric and stationary axisymmetric spacetimes.

\section{B. General static spherically symmetric spacetimes}

We take the ansatz,

$$
d s^{2}=f(r) d t^{2}-h(r) d r^{2}-r^{2} d \Omega^{2} .
$$

We assume that the above metric admits a cosmological event horizon, located (in a dimensionless unit as earlier) at $r=1$,

$$
f(r \rightarrow 1) \rightarrow 0, \quad h^{-1}(r \rightarrow 1) \rightarrow 0,
$$

with $f h(r \rightarrow 1)$ being neither vanishing nor divergent. Equation (40) can represent, e.g., the Schwarzschild-de Sitter spacetime [i.e., $f(r)=\left(1-2 M / r-H_{0}^{2} r^{2}\right)=h^{-1}(r)$ ] or its charged variants.

The existence of the Killing horizon guarantees the existence of causally disconnected regions like $\mathrm{R}$ and $\mathrm{L}$ similar to Fig. 1. The tortoise coordinate is defined in this case as

$$
r_{\star}:=\int d r \sqrt{\frac{h}{f}},
$$

whereas the Kruskal null coordinates at the cosmological event horizon will similar to that of Sec. II. Thus, as of Sec. III A, due to the spherical symmetry, we can look for a solution of the form

$$
\Psi=\left(\begin{array}{c}
\Psi_{1} \\
\Psi_{2}
\end{array}\right)=\frac{e^{-i p t}}{r f^{\frac{1}{4}}}\left(\begin{array}{c}
i \tilde{\Psi}_{1}(r) \Omega_{l+1 / 2, l, m} \\
\tilde{\Psi}_{2}(r) \Omega_{(l+1)-1 / 2, l+1, m}
\end{array}\right)
$$

so that we obtain

$$
\begin{gathered}
\left(-\frac{p}{\sqrt{f}}+m_{0}\right) \tilde{\Psi}_{1}+\left(\frac{1}{\sqrt{h}} \partial_{r}+\frac{h^{-\frac{1}{2}}}{r}+\frac{(l+1)}{r}\right) \tilde{\Psi}_{2}=0 \\
\left(\frac{p}{\sqrt{f}}+m_{0}\right) \tilde{\Psi}_{2}+\left(\frac{1}{\sqrt{h}} \partial_{r}+\frac{h^{-\frac{1}{2}}}{r}-\frac{(l+1)}{r}\right) \tilde{\Psi}_{1}=0 .
\end{gathered}
$$

To the best of our knowledge, unlike the static de Sitter, the Dirac equation cannot be solved in a closed form, even in the Schwarzschild-de Sitter background. However, we recall from the preceding section that in order to study the entanglement we need the Bogoliubov relations between the local R-L modes and the global ones. To find the global modes, on the other hand, we must analytically continue the local modes across the horizon. Thus, we need to be concerned only about the near-horizon forms of the modes.
The near-horizon limit of Eq. (42) after using Eq. (41) is given by

$$
\partial_{r_{\star}} \tilde{\Psi}_{2}=p \tilde{\Psi}_{1}, \quad \partial_{r_{\star}} \tilde{\Psi}_{1}=-p \tilde{\Psi}_{2},
$$

which yield modes similar to Eq. (24) and Eq. (25). By defining the Kruskal null coordinates analogously as the static de Sitter, we can find out the global modes similar to Eq. (26) and Eq. (27). Hence, we shall obtain identical Bogoliubov relations as Eq. (31) and the entanglement entropy, Eq. (39).

Note also that Eq. (40) in addition can also possess a black hole event horizon, located inside the cosmological event horizon. As long as the black hole horizon is nonextremal, the computation of the entanglement entropy for the black hole will be similar to that of the cosmological horizon. One needs two different sets of Kruskal coordinates to analyse the near-horizon modes.

We shall end this section with comment on the Nariai limit of the Schwarzschild-de Sitter spacetime $\left(3 \sqrt{3} M H_{0} \rightarrow 1\right)$, for which the radial values of the cosmological and black hole event horizons are nearly coincident. This makes the proper separation between them large. Utilizing this proper separation as a coordinate, the metric can be written as, e.g., [10],

$$
d s^{2}=\frac{1}{9 H_{0}^{4}}\left[\frac{1}{\cosh ^{2} x}\left(d t^{2}-d x^{2}\right)-d \Omega^{2}\right] .
$$

The above metric is $d S^{2} \times S^{2}$ and is endowed with a black hole and cosmological event horizon, located, respectively, at $x \rightarrow \mp \infty$. Result similar to Eq. (39) follows for this case as well.

\section{STATIONARY AXISYMMETRIC SPACETIMES}

We finally come to the case of the stationary axisymmetric spacetimes endowed with a positive $\Lambda$. For example, the Kerr-de Sitter spacetime in the Boyer-Lindquist coordinates reads

$$
\begin{aligned}
d s^{2}= & \frac{\Delta_{r}-a^{2} \sin ^{2} \theta \Delta_{\theta}}{\rho^{2}} d t^{2}+\frac{2 a \sin ^{2} \theta}{\rho^{2} \Xi}\left(\left(r^{2}+a^{2}\right) \Delta_{\theta}-\Delta_{r}\right) d t d \phi \\
& -\frac{\sin ^{2} \theta}{\rho^{2} \Xi^{2}}\left(\left(r^{2}+a^{2}\right)^{2} \Delta_{\theta}-\Delta_{r} a^{2} \sin ^{2} \theta\right) d \phi^{2} \\
& -\frac{\rho^{2}}{\Delta_{r}} d r^{2}-\frac{\rho^{2}}{\Delta_{\theta}} d \theta^{2}
\end{aligned}
$$

where

$$
\begin{aligned}
\Delta_{r} & =\left(r^{2}+a^{2}\right)\left(1-H_{0}^{2} r^{2}\right)-2 M r, \quad \Delta_{\theta}=1+H_{0}^{2} a^{2} \cos ^{2} \theta, \\
\Xi & =1+H_{0}^{2} a^{2}, \quad \rho^{2}=r^{2}+a^{2} \cos ^{2} \theta,
\end{aligned}
$$

with $H_{0}^{2}=\Lambda / 3$ as earlier. The parameter $a$ is related to the spacetime rotation. For $a=0$, we recover the Schwarzschild-de Sitter spacetime, whereas setting 
$M=0$, we obtain the de Sitter spacetime written in the static patch.

Unlike the previous cases, we shall keep the parameters intact, for the purpose of some numerical analyses we wish to perform. The cosmological event horizon is given by the largest root of $\Delta_{r}=0$. Its surface gravity is given by

$$
-\kappa_{C}=\left.\frac{\Delta_{r}^{\prime}}{2\left(r^{2}+a^{2}\right)}\right|_{r=r_{C}}
$$

with $\kappa_{C}>0$. Finally, the horizon Killing field is given by $\chi_{C}=\partial_{t}+\Omega_{C} \partial_{\phi}$, with

$$
\Omega_{C}=\frac{a \Xi}{r_{C}^{2}+a^{2}}
$$

being the angular speed on the cosmological event horizon. There can be a black hole horizon at $r=r_{H}\left(r_{H} \leq r_{C}\right)$, as well.

Aspects of quantum entanglement in the Kerr spacetime for a scalar field with its various vacuum states can be seen in Ref. [51]. Because of the existence of the ergosphere, the timelike Killing vector field in rotating spacetimes like Eq. (45) becomes spacelike on or in the neighborhood of the horizon. To tackle this difficulty, usually one needs to consider the horizon Killing vector field, future directed and null on the horizon. This vector field describes a family of observers corotating with the same angular speed on the horizon. Aspects of quantization of fermions in rotating backgrounds with the vacuum state defined with respect to such rigidly rotating observers can be seen in Refs. [52-55].

Now, the Dirac equation in Eq. (45) can be studied using the usual Newman-Penrose basis, e.g., Ref. [56] and references therein. However, to define the aforementioned rigidly rotating states near the horizon, it is a bit convenient to go to a diagonal basis as follows. Let us define a vector field,

$$
\chi^{\mu}=\left(\partial_{t}\right)^{\mu}-\frac{\left(\partial_{t} \cdot \partial_{\phi}\right)}{\left(\partial_{\phi} \cdot \partial_{\phi}\right)}\left(\partial_{\phi}\right)^{\mu}=\left(\partial_{t}\right)^{\mu}-\frac{g_{t \phi}}{g_{\phi \phi}}\left(\partial_{\phi}\right)^{\mu} .
$$

It satisfies $\chi \cdot \partial_{\phi}=0$ and also trivially $\chi \cdot \partial_{r}=0=\chi \cdot \partial_{\theta}$. We have

$\chi^{\mu} \chi_{\mu}=\frac{g_{t t} g_{\phi \phi}-g_{t \phi}^{2}}{g_{\phi \phi}}=\frac{\rho^{2} \Delta_{r} \Delta_{\theta}}{\left(r^{2}+a^{2}\right)^{2} \Delta_{\theta}-\Delta_{r} a^{2} \sin ^{2} \theta}=\beta^{2}$ (say),

which, with $\Delta_{r}>0$, is easily seen to be positive. In other words, $\chi^{\mu}$ is a timelike vector field, and it is null on the horizon(s), $\Delta_{r}=0$. It can be also seen that a) $\chi^{\mu}$ satisfies the Frobenius condition of hypersurface orthogonality and, b) even though $\chi^{\mu}$ is not in general Killing, it smoothly coincides with the horizon Killing field(s) [57]. Thus, we can define the following orthonormal basis for Eq. (45):

$$
e_{0}^{\mu}=\beta^{-1} \chi^{\mu}, \quad e_{1}^{\mu}=\frac{1}{\sqrt{-g_{r r}}}\left(\partial_{r}\right)^{\mu}, \quad e_{2}^{\mu}=\frac{1}{\sqrt{-g_{\theta \theta}}}\left(\partial_{\theta}\right)^{\mu}, \quad e_{3}^{\mu}=\frac{1}{\sqrt{-g_{\phi \phi}}}\left(\partial_{\phi}\right)^{\mu} .
$$

With this choice and the representation of the $\gamma$-matrices mentioned in Sec. III, we expand Eq. (6). For the positive frequency modes, we take the ansatz $\Psi=e^{-i p t+i m \phi} \tilde{\Psi}(r, \theta)$. As earlier, we focus only on the near-horizon limit, $\Delta_{r} \rightarrow 0$, to obtain

$$
\begin{aligned}
& \frac{\left(r^{2}+a^{2}\right)}{\rho \sqrt{\Delta_{r}}} \gamma^{0}\left(p-m \Omega_{C}\right) \tilde{\Psi}+\frac{i \sqrt{\Delta_{r}}}{\rho} \gamma^{1} \partial_{r} \tilde{\Psi}+\frac{i \sqrt{\Delta_{\theta}}}{\rho} \gamma^{2} \partial_{\theta} \tilde{\Psi}+\frac{i \rho \Xi}{\left(r^{2}+a^{2}\right) \sin \theta \sqrt{\Delta_{\theta}}} \gamma^{3} \partial_{\phi} \tilde{\Psi} \\
& \quad+\frac{i}{4}\left[\frac{\Delta_{r}^{\prime}}{\rho \sqrt{\Delta_{r}}} \gamma^{1} \gamma^{2}+\frac{\rho \partial_{\theta}\left(\Delta_{\theta} \sin ^{2} \theta / \rho^{2}\right)}{\sin ^{2} \theta \sqrt{\Delta_{\theta}}}\right] \gamma^{2} \tilde{\Psi}-m \tilde{\Psi}+\mathcal{O}\left(\Delta_{r}^{2}\right)=0,
\end{aligned}
$$

We multiply both sides with $\rho \sqrt{\Delta_{r}} /\left(r^{2}+a^{2}\right)$ and rewrite it using the tortoise coordinate defined as

$$
d r_{\star}=\int \frac{\left(r^{2}+a^{2}\right)}{\Delta_{r}} .
$$

Note that as $r \rightarrow r_{C}$ or $\Delta_{r} \rightarrow 0$ various terms, including the mass term, drop. We find for the two positive frequency nearhorizon modes in the region $\mathrm{R}$

$$
\Psi_{p \lambda m}^{R 1+}=\frac{\sqrt{\Xi}}{\sqrt{2 \pi \rho} \Delta_{r}^{\frac{1}{4}}} e^{-i\left(p-m \Omega_{C}\right) v_{R}+i m \phi_{C}}\left(\begin{array}{c}
S_{+}(\lambda, \theta) \\
S_{-}(\lambda, \theta) \\
-S_{-}(\lambda, \theta) \\
S_{+}(\lambda, \theta)
\end{array}\right), \quad \Psi_{p \lambda m}^{R 2+}=\frac{\sqrt{\Xi}}{\sqrt{2 \pi \rho} \Delta_{r}^{\frac{1}{4}}} e^{-i\left(p-m \Omega_{C}\right) v_{R}+i m \phi_{C}}\left(\begin{array}{c}
S_{+}(\lambda, \theta) \\
S_{-}(\lambda, \theta) \\
S_{-}(\lambda, \theta) \\
-S_{+}(\lambda, \theta)
\end{array}\right),
$$




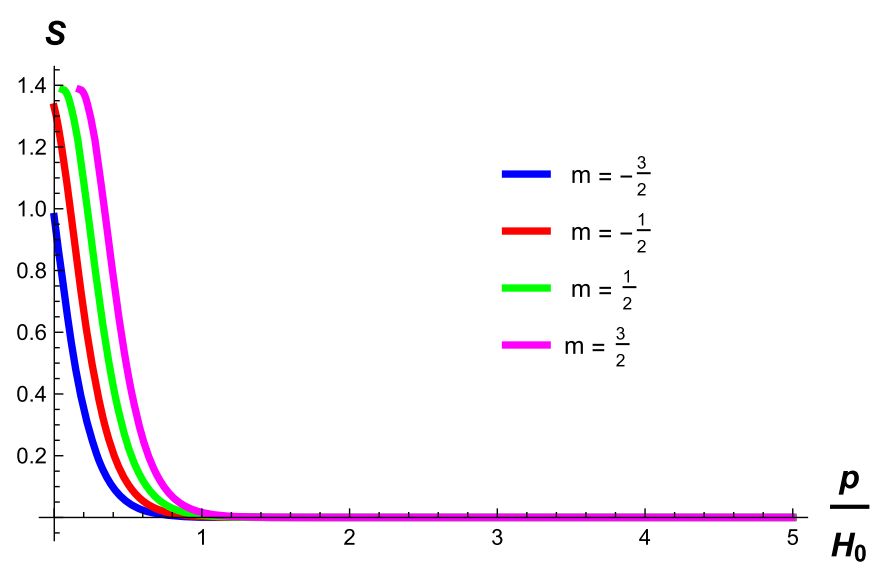

(a) $a H_{0}=0.09, M H_{0}=0.1$
$\mathbf{S}$

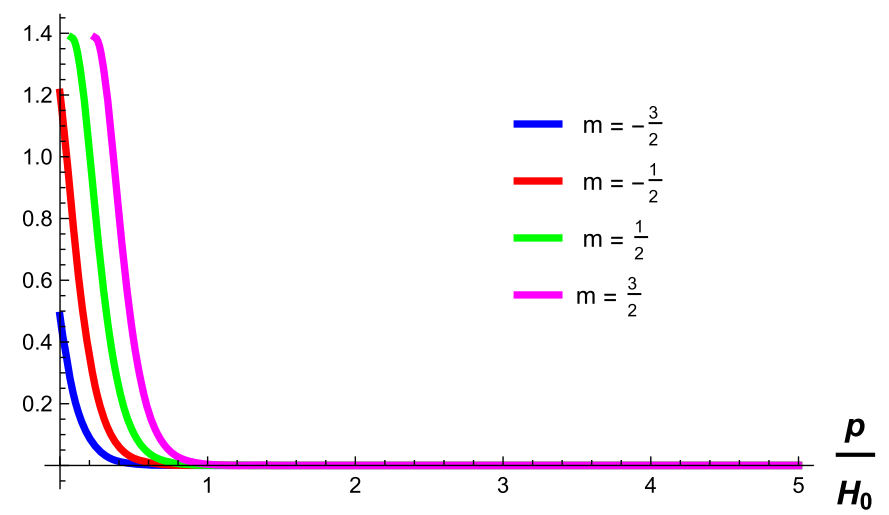

(b) $a H_{0}=0.1, M H_{0}=0.15$

FIG. 2. The variation of the entanglement entropy, Eq. (49), with respect to the dimensionless energy $p / H_{0}$, with different angular eigenvalues.

where the untwisted azimuthal coordinate $\phi_{C}:=\phi-\Omega_{C} t$ defines a rigidly rotating observer on the horizon and $v=$ $t+r_{\star}$ as earlier. The negative frequency modes are found via the charge conjugation of Eq. (48). The modes in the $\mathrm{L}$ region are also found by making the time past directed, as in Eq. (25). Note that in order to have any sensible field quantization we must have $p-m \Omega_{C} \geq 0$.

The angular functions $S_{ \pm}(\lambda, \theta)$ are spin- $1 / 2$ weighted spheroidal harmonics with eigenvalues $\lambda$, e.g., Ref. [56] and references therein. They are normalized as

$$
\int_{0}^{\pi} d \theta \sin \theta\left[S_{+}^{\star}(\lambda, \theta) S_{+}\left(\lambda^{\prime}, \theta\right)+S_{-}^{\star}(\lambda, \theta) S_{-}\left(\lambda^{\prime}, \theta\right)\right]=\delta_{\lambda \lambda^{\prime}}
$$

We shall not require their explicit forms for our current purpose.

Choosing our integration hypersurface to be orthogonal to the vector field $\chi^{\mu}$, it can be easily checked as earlier that the modes of Eq. (48) (along with the negative frequency

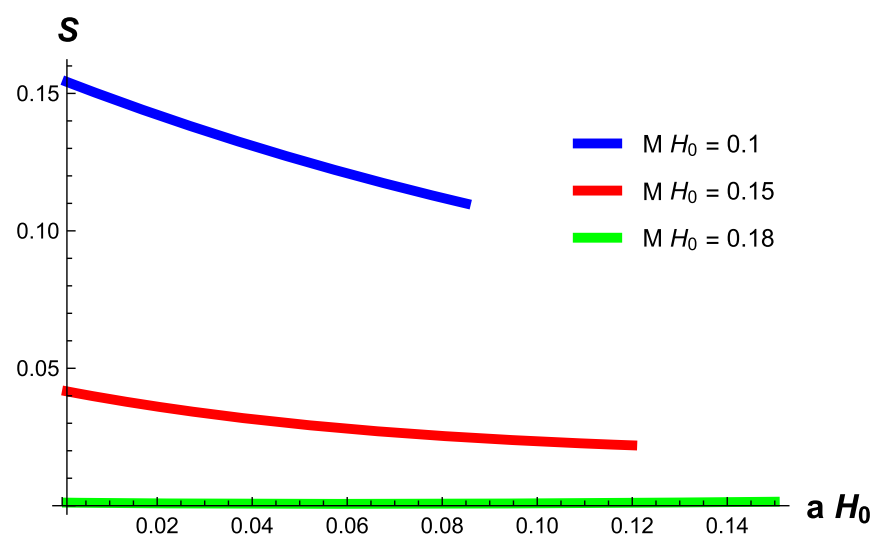

(a) $\frac{p}{H_{0}}=0.5, m=-1 / 2$ modes) are $\delta$-function normalizable and they form an orthonormal set. With this, we now perform the same analysis as described in Sec. IV A to obtain a generalization of Eq. (39),

$S(p, m)=2 \ln \left(1+e^{-\frac{2 \pi\left(p-m \Omega_{C}\right)}{\kappa_{C}}}\right)+\frac{4 \pi\left(p-m \Omega_{C}\right)}{\kappa_{C}\left(1+e^{\frac{2 \pi\left(p-m \Omega_{C}\right)}{{ }^{K_{C}}}}\right)}$

Formally, a similar expression corresponding to the black hole event horizon is obtained simply by replacing $\kappa_{C}$ by $\kappa_{H}$ and $\Omega_{C}$ by $\Omega_{H}$, respectively the surface gravity and the angular speed of the black hole horizon. Setting $\Omega_{C}=0$ in the above equation yields the result for the Schwarzschildde Sitter spacetime. Setting further $M=0$ recovers Eq. (39), with the dimensionless scaling $p \rightarrow p / \kappa_{C}$.

We now wish to numerically investigate the variation of $S(p, m)$ in Eq. (49), with respect to $p, m$, and the spacetime parameters. If we assume a Kerr-de Sitter black hole

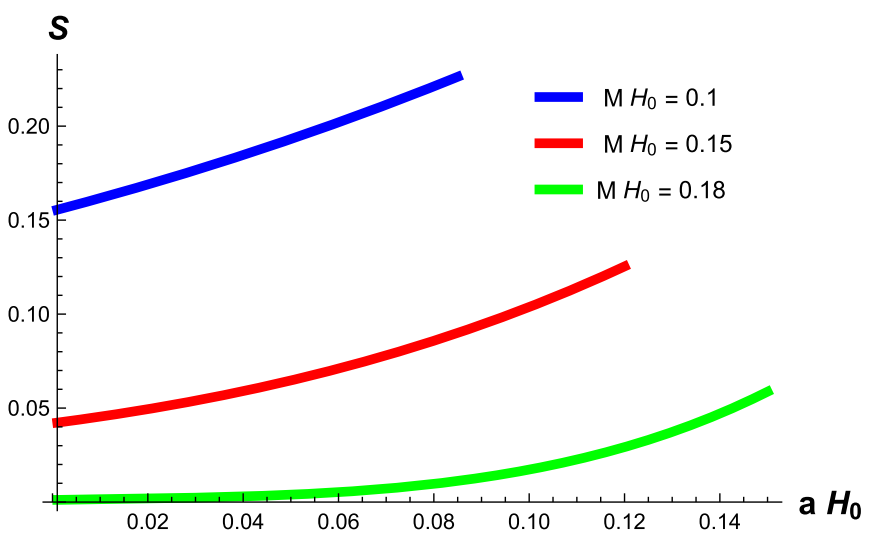

(b) $\frac{p}{H_{0}}=0.5, m=1 / 2$

FIG. 3. The variation of the entanglement entropy, Eq. (49), with respect to the dimensionless rotation parameter, $a H_{0}$. The qualitative difference of the $m>0$ and $m<0$ states is manifest here. 


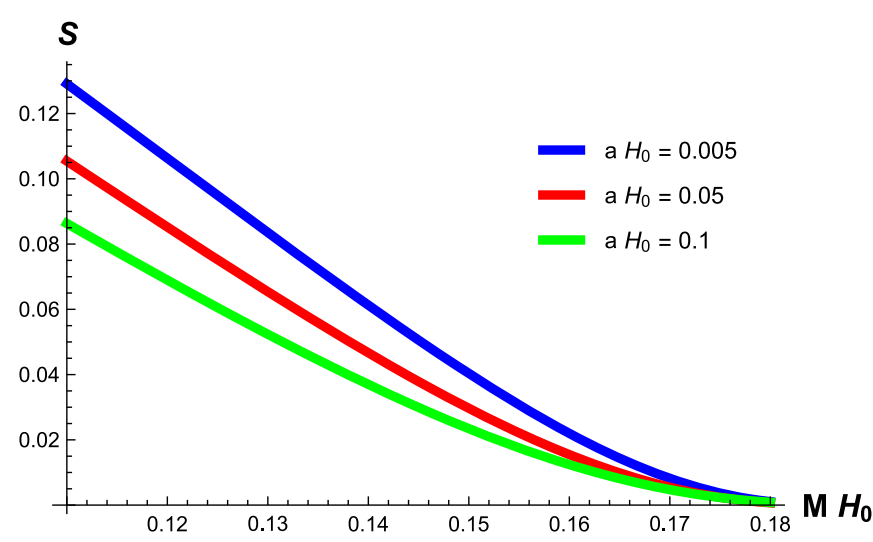

(a) $\frac{p}{H_{0}}=0.5, m=-1 / 2$

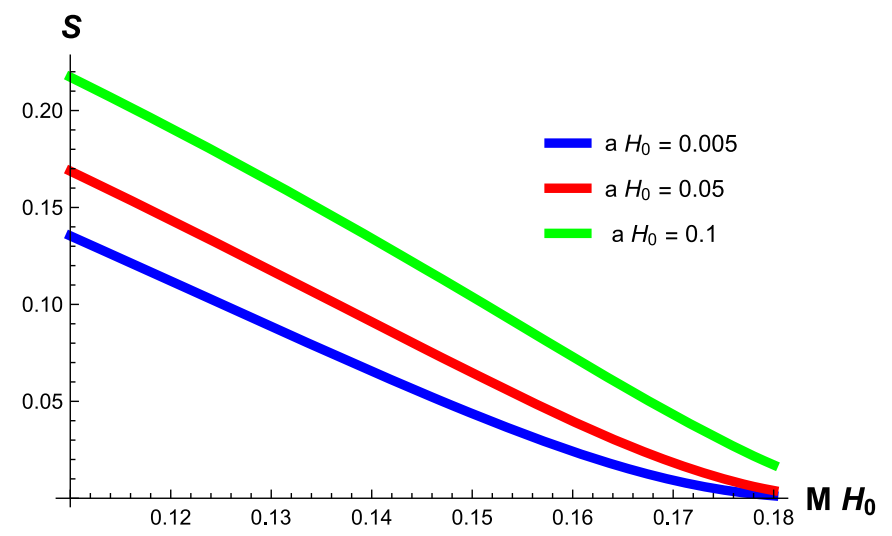

(b) $\frac{p}{H_{0}}=0.5, m=1 / 2$

FIG. 4. The variation of the entanglement entropy, Eq. (49), with respect to the dimensionless mass parameter, $M H_{0}$.

spacetime, we have the bounds on the dimensionless parameters, $M H_{0} \lesssim 0.2435$ and $a / M \lesssim 1$, e.g., Ref. [58]. Figure 2 shows the monotonic decrease of $S(p, m)$ with respect to the increase of the dimensionless energy, $p / H_{0}$. Figures 3 and 4 show the variation of $S(p, m)$, respectively, with respect to the dimensionless rotation and mass parameters. In Fig. 3, the distinction of the positive and negative $m$ values are manifest, following from the increase of both $\Omega_{C}$ and $\kappa_{C}$ with the increase of $a H_{0}$. This distinction is a quantum analog of the distinction of the classical pro- and retrograde orbits [43]. The decrease of $S(p, m)$ with respect to increasing $M H_{0}$ corresponds to the fact that $\kappa_{C}$ decreases, whereas $\Omega_{C}$ increases with the increase of the same.
Finally, we note that, even though for the black hole event horizon Eq. (49) is formally similar, its horizon parameters, $\kappa_{H}, \Omega_{H}$ show some qualitatively different variations with respect to $a H_{0}$ and $M H_{0}$. Thus, in this case, while Fig. 2 will remain similar, a priori, one might expect Fig. 3 and Fig. 4 to show different behavior. However, this is not the case, as can be checked numerically. In fact the variation of the term $\left(p-m \Omega_{H}\right) / \kappa_{H}$ is such that the entanglement entropy for the black hole horizon behaves qualitatively similarly to that of the cosmological horizon.

The above result can be extended to more general stationary axisymmetric spacetimes, for example, the general Plebanski-Demianski-de Sitter class [59],

$$
d s^{2}=\frac{1}{\Omega^{2}}\left[-\frac{\Delta_{r}}{\rho^{2}}\left(d t-\left(a \sin ^{2} \theta+4 l \sin ^{2} \frac{\theta}{2}\right) d \phi\right)^{2}+\frac{\rho^{2}}{\Delta_{r}} d r^{2}+\frac{P}{\rho^{2}}\left(a d t-\left(r^{2}+(a+l)^{2}\right) d \phi\right)^{2}+\frac{\rho^{2}}{P} \sin ^{2} \theta d \theta^{2}\right],
$$

where

$$
\begin{aligned}
& \Omega=1-\frac{\alpha}{\omega}(l+a \cos \theta) r, \quad \rho^{2}=r^{2}+(l+a \cos \theta)^{2}, \quad P=\sin ^{2} \theta\left(1-a_{3} \cos \theta-a_{4} \cos ^{2} \theta\right) \\
& \Delta_{r}=\left(\omega^{2} k+q^{2}+q_{m}^{2}\right)-2 M r+\epsilon r^{2}-\frac{2 \alpha n}{\omega} r^{3}-\left(\alpha^{2} k+H_{0}^{2}\right) r^{4} .
\end{aligned}
$$

The parameters $\alpha, \omega, q, q_{m}, \epsilon$, and $k$ are independent, and $a_{3}$ and $a_{4}$ are determined from them via a couple of constraints. The physical meaning of these parameters could be asserted for only certain special subclasses of Eq. (50). For example, for $\alpha=0$, the above metric reduces to the Kerr-Newman-NUT-de Sitter solution [59],

$$
d s^{2}=-\frac{\Delta_{r}}{\rho^{2}}\left[d t-\left(a \sin ^{2} \theta+4 l \sin ^{2} \frac{\theta}{2}\right) d \phi\right]^{2}+\frac{\rho^{2}}{\Delta_{r}} d r^{2}+\frac{P}{\rho^{2}}\left[a d t-\left(r^{2}+(a+l)^{2}\right) d \phi\right]^{2}+\frac{\rho^{2}}{P} \sin ^{2} \theta d \theta^{2},
$$

where

$$
\begin{aligned}
& \rho^{2}=r^{2}+(l+a \cos \theta)^{2}, \quad P=\sin ^{2} \theta\left(1+4 a l H_{0}^{2} \cos \theta+H_{0}^{2} a^{2} \cos ^{2} \theta\right) \\
& \Delta_{r}=\left(a^{2}-l^{2}+q^{2}+q_{m}^{2}\right)-2 M r+r^{2}-3 H_{0}^{2}\left(\left(a^{2}-l^{2}\right) l^{2}+\left(\frac{a^{2}}{3}+2 l^{2}\right) r^{2}+\frac{r^{4}}{3}\right),
\end{aligned}
$$


where $q$ and $q_{m}$ are, respectively, the electric and magnetic charge and $l$ is the NUT parameter. We shall consider below the most general form of Eq. (50), assuming implicitly it indeed represents a well-behaved spacetime possessing a cosmological event horizon at $\Delta_{r}=0$. We shall not be concerned with the explicit parameter values corresponding to this assertion.

The choice of the hypersurface orthogonal timelike vector field $\chi^{\mu}$ and the orthogonal basis for this case is exactly formally similar to the Kerr-de Sitter spacetime described above. Likewise, the R-L entanglement entropy turns out to be formally similar to Eq. (49) in this case, with the horizon parameters

$$
\Omega_{C}=\frac{a}{r_{C}^{2}+(a+l)^{2}} \quad-\kappa_{C}=\left.\frac{\Delta_{r}^{\prime}}{2\left(r^{2}+a^{2}\right)}\right|_{r=r_{C}} .
$$

\section{CONCLUSIONS}

In this work, we have addressed the issue of the quantum entanglement for the Dirac fermions between the causally disconnected R-L regions of a static de Sitter spacetime. We have discussed the case of the simple de Sitter spacetime using the closed form mode functions. The behavior of the fermionic entanglement entropy, like that of a scalar field [38], was shown to be similar to that of the Rindler spacetime $[39,40]$. We further extended our result to the case of general static and spherically symmetric spacetime as well as to the stationary axisymmetric spacetimes. For the Kerr-de Sitter spacetime, in particular, we have numerically investigated the variation of the entanglement entropy with respect to the energy and angular momentum eigenvalues as well as the (dimensionless) spacetime parameters. The entanglement entropy for the nonextremal black hole horizon also shows similar variations with respect to these parameters.

An interesting direction in which this work can be extended seems to be the consideration of charged fields in the presence of background electromagnetic fields associated with a Killing horizon. We hope to come back to this issue in the near future.

\section{ACKNOWLEDGMENTS}

S. G. would like to acknowledge I. Cotaescu and A. Abrikosov, Jr., for helpful discussions. S. B.'s research is partially supported by the ISIRD Grant No. 9-289/2017/ IITRPR/704. S. C. is partially supported by the ISIRD Grant No. 9-252/2016/IITRPR/708.
[1] S. Weinberg, Cosmology (Oxford University, New York, 2008).

[2] T. S. Bunch and P. C. W. Davies, Quantum field theory in de Sitter space: Renormalization by point splitting, Proc. Roy. Soc. A 360, 117 (1978).

[3] E. Mottola, Particle creation in de Sitter space, Phys. Rev. D 31, 754 (1985).

[4] B. Allen, Vacuum states in de Sitter space, Phys. Rev. D 32, 3136 (1985).

[5] M. B. Fröb, J. Garriga, S. Kanno, M. Sasaki, J. Soda, T. Tanaka, and A. Vilenkin, Schwinger effect in de Sitter space, J. Cosmol. Astropart. Phys. 04 (2014) 009.

[6] G. W. Gibbons and S. W. Hawking, Cosmological event horizons, thermodynamics, and particle creation, Phys. Rev. D 15, 2738 (1977).

[7] V.S. Otchik, On the hawking radiation of spin $1 / 2$ particles in the de sitter space-time, Classical Quantum Gravity 2, 539 (1985).

[8] A. Higuchi, Quantization of scalar and vector fields inside the cosmological event horizon and its application to hawking effect, Classical Quantum Gravity 4, 721 (1987).

[9] R. Bousso and S. W. Hawking, (Anti)evaporation of Schwarzschild-de Sitter black holes, Phys. Rev. D 57, 2436 (1998).
[10] S. Nojiri and S. D. Odintsov, Anti-evaporation of Schwarzschild-de Sitter black holes in $F(R)$ gravity, Classical Quantum Gravity 30, 125003 (2013).

[11] Y. Qiu and J. Traschen, Black hole and cosmological particle production in Schwarzschild de Sitter, arXiv: 1908.02737.

[12] R. P. Woodard, Perturbative quantum gravity comes of age, Int. J. Mod. Phys. D 23, 1430020 (2014).

[13] S. P. Miao, T. Prokopec, and R. P. Woodard, Scalar enhancement of the photon electric field by the tail of the graviton propagator, Phys. Rev. D 98, 025022 (2018).

[14] G. Moreau and J. Serreau, Stability of de Sitter Spacetime Against Infrared Quantum Scalar Field Fluctuations, Phys. Rev. Lett. 122, 011302 (2019).

[15] D. Benisty, E. I. Guendelman, E. N. Saridakis, H. Stoecker, J. Struckmeier, and D. Vasak, Inflation from fermions with curvature-dependent mass, Phys. Rev. D 100, 043523 (2019).

[16] D. Benisty, Inflation from fermions, arXiv:1912.11124.

[17] J. Maldacena and G. L. Pimentel, Entanglement entropy in de Sitter space, J. High Energy Phys. 02 (2013) 038.

[18] S. Kanno, M. Sasaki, and T. Tanaka, Vacuum state of the dirac field in de Sitter space and entanglement entropy, J. High Energy Phys. 03 (2017) 068.

[19] S. Bhattacharya, S. Chakrabortty, and S. Goyal, Emergent $\alpha$-like fermionic vacuum structure and entanglement in the 
hyperbolic de Sitter spacetime, Eur. Phys. J. C 79, 799 (2019).

[20] S. Kanno, J. Murugan, J. P. Shock, and J. Soda, Entanglement entropy of $\alpha$-vacua in de Sitter space, J. High Energy Phys. 07 (2014) 072.

[21] N. Iizuka, T. Noumi, and N. Ogawa, Entanglement entropy of de Sitter space $\alpha$-vacua, Nucl. Phys. B910, 23 (2016).

[22] S. Kanno, Impact of quantum entanglement on spectrum of cosmological fluctuations, J. Cosmol. Astropart. Phys. 07 (2014) 029.

[23] S. Kanno, Cosmological implications of quantum entanglement in the multiverse, Phys. Lett. B 751, 316 (2015).

[24] S. Kanno, A note on initial state entanglement in inflationary cosmology, Europhys. Lett. 111, 60007 (2015).

[25] S. Choudhury, S. Panda, and R. Singh, Bell violation in the Sky, Eur. Phys. J. C 77, 60 (2017).

[26] S. Choudhury, S. Panda, and R. Singh, Bell violation in primordial cosmology, Universe 3, 13 (2017).

[27] S. Kanno and J. Soda, Infinite violation of Bell inequalities in inflation, Phys. Rev. D 96, 083501 (2017).

[28] S. Kanno, J. P. Shock, and J. Soda, Quantum discord in de Sitter space, Phys. Rev. D 94, 125014 (2016).

[29] A. Albrecht, S. Kanno, and M. Sasaki, Quantum entanglement in de Sitter space with a wall, and the decoherence of bubble universes, Phys. Rev. D 97, 083520 (2018).

[30] S. Choudhury, A. Mukherjee, P. Chauhan, and S. Bhattacherjee, Quantum Out-of-Equilibrium Cosmology, Eur. Phys. J. C 79, 320 (2019).

[31] J. Feng, X. Huang, Y. Z. Zhang, and H. Fan, Bell inequalities violation within non-Bunch-Davies states, Phys. Lett. B 786, 403 (2018).

[32] S. Yamaguchi, R. Tatsukawa, S. Y. Lin, and K. Yamamoto, Late-time quantum radiation by a uniformly accelerated detector in de Sitter spacetime, Phys. Rev. D 98, 105012 (2018).

[33] G. Narain and H. Q. Zhang, Non-locality effect on the entanglement entropy in de Sitter, J. Cosmol. Astropart. Phys. 06 (2019) 012.

[34] X. Dong, E. Silverstein, and G. Torroba, De Sitter holography and entanglement entropy, J. High Energy Phys. 07 (2018) 050.

[35] C. Arias, F. Diaz, and P. Sundell, De Sitter space and entanglement, Classical Quantum Gravity 37, 015009 (2020).

[36] H. Geng, S. Grieninger, and A. Karch, Entropy, entanglement and swampland bounds in DS/dS, J. High Energy Phys. 06 (2019) 105.

[37] H. Geng, Some information theoretic aspects of de-Sitter holography, J. High Energy Phys. 02 (2020) 005.

[38] A. Higuchi and K. Yamamoto, Vacuum state in de Sitter spacetime with static charts, Phys. Rev. D 98, 065014 (2018).
[39] P. M. Alsing, I. Fuentes-Schuller, R. B. Mann, and T. E. Tessier, Entanglement of Dirac fields in non-inertial frames, Phys. Rev. A 74, 032326 (2006).

[40] D. C. M. Ostapchuk and R. B. Mann, Generating entangled fermions by accelerated measurements on the vacuum, Phys. Rev. A 79, 042333 (2009).

[41] A. Lopez-Ortega, Absorption and quasinormal modes of classical fields propagating on 3D and 4D de Sitter spacetime, Gen. Relativ. Gravit. 38, 743 (2006).

[42] A. Lopez-Ortega, Dirac quasinormal modes of Ddimensional de Sitter spacetime, Gen. Relativ. Gravit. 39, 1011 (2007).

[43] S. Chandrasekhar, The Mathematical Theory of Black Holes (Oxford University, Oxford, England, 1983).

[44] I. I. Cotaescu, The Dirac particle on de Sitter background, Mod. Phys. Lett. A 13, 2991 (1998).

[45] I. I. Cotaescu, Dirac fermions in de Sitter and anti-de Sitter backgrounds, Rom. J. Phys. 52, 895 (2007).

[46] D. R. Brill and J. A. Wheeler, Interaction of neutrinos and gravitational fields, Rev. Mod. Phys. 29, 465 (1957).

[47] V. B. Berestetskii, E. M. Lifshitz, and L. P. Pitaevskii, Relativistic Quantum Theory: Part I (Pergamon, New York, 1971).

[48] M. Abramowitz and I. A. Stegun, Handbook of Mathematical Functions with Formulas, Graphs, and Mathematical Tables, National Bureau of Standards (Dover Publications, INC., New York, 1972).

[49] W. G. Unruh, Notes on black hole evaporation, Phys. Rev. D 14, 870 (1976).

[50] M. Socolovsky, Rindler space and unruh effect, arXiv: 1304.2833.

[51] G. Menezes, Entanglement dynamics in a Kerr spacetime, Phys. Rev. D 97, 085021 (2018).

[52] M. Casals, S. R. Dolan, B. C. Nolan, A. C. Ottewill, and E. Winstanley, Quantization of fermions on Kerr space-time, Phys. Rev. D 87, 064027 (2013).

[53] V.E. Ambrus and E. Winstanley, Rotating quantum states, Phys. Lett. B 734, 296 (2014).

[54] A. Belokogne and A. Folacci, Renormalized stress tensor for massive fields in Kerr-Newman spacetime, Phys. Rev. D 90, 044045 (2014).

[55] G. Z. Toth, Weak cosmic censorship, dyonic Kerr-Newman black holes and Dirac fields, Classical Quantum Gravity 33, 115012 (2016).

[56] D. Batic, K. Morgan, M. Nowakowski, and S. B. Medina, The Dirac equation in the Kerr-de Sitter metric, Gravitation Cosmol. 24, 224 (2018).

[57] S. Bhattacharya and A. Lahiri, No hair theorems for stationary axisymmetric black holes, Phys. Rev. D 83, 124017 (2011).

[58] S. Bhattacharya, Kerr-de Sitter spacetime, Penrose process and the generalized area theorem, Phys. Rev. D 97, 084049 (2018).

[59] J. B. Griffiths and J. Podolsky, A New look at the PlebanskiDemianski family of solutions, Int. J. Mod. Phys. D 15, 335 (2006). 